\title{
Linajes budistas en Uruguay
}

\author{
Ismael ApUd, Mauro Clara y Paul RuIZ \\ Universidad de la República (Uruguay) \\ ismaelapud@psico.edu.uy / primacruzada@gmail.com / paulruiz@psico.edu.uy
}

\begin{abstract}
RESUMEN
La llegada del budismo al Uruguay se encuentra relacionada a varios factores histórico-culturales, cómo ser la apertura democrática de los ochenta, la progresiva globalización del país, la ruptura posmoderna con un modelo hegemónico racionalista-positivista, la emergencia de los denominados Nuevos Movimientos Religiosos y la apertura a mercados de bienes simbólico-religiosos, generalmente dirigidos a estratos sociales medios y altos, con capital económico y cultural propicio para el consumo de propuestas existenciales e introspectivas. En el siguiente trabajo nos proponemos analizar la llegada de los distintos linajes budistas en Uruguay (tibetanos y japoneses), bajo el contexto histórico y socio-cultural que habilita su emergencia.
\end{abstract}

Palabras clave: budismo, Uruguay, religión, posmodernidad, secularización.

\section{Buddhist lineage in Uruguay}

\begin{abstract}
The arrival of Buddhism to Uruguay is related to various historical and cultural factors, such as the democratic opening at eighties, the progressive globalization in the country, the break of post (modernism) with a hegemonic rationalist-positivist model, the emergence of so-called New Religious Movements and opening of goods symbolic-religious markets, generally aimed at middle and upper social strata with economic and cultural capital is conducive to the proposed use of existential and introspective way of life. In this paper we analyze the arrival of the different Buddhist lineages in Uruguay (Tibetan and Japanese), and the historical and socio-cultural context which activates their emergency.
\end{abstract}

Keywords: Buddhism, Uruguay, religion, postmodernity, secularization.

SUMARIO: 1. Introducción. 2. Trazos de una genealogía. 3. Budismo tibetano. 3.1.Diamond Way. 3.2. La Chagdud Gonpa. 4. Budismo Zen. 5. Budismo Nichiren. 5.1.Soka Gakkai. 5.2.La Nichiren Shu. 6. Otros movimientos relacionados con el budismo. 7. Conclusiones. 


\section{INTRODUCCIÓN}

El proceso histórico de consolidación del Estado moderno en Uruguay tiene fuertes lazos ideológicos con el republicanismo francés. A principios del siglo XX ya vemos claramente cómo las ideas jacobinas y liberales prevalecen en los campos de la política y la economía nacional. En las primeras décadas del siglo éstas se retroalimentan del movimiento positivista y comienza a imponerse con fuerza la idea de separación entre instituciones políticas y religiosas ${ }^{1}$. Este proceso que, en sentido weberiano llamamos de secularización, obtiene status oficial con la aprobación del Art. $5^{\circ}$ de la Constitución de la República de 1917. Bajo esta ideología se dan forma y legitiman las diversas instituciones del Estado (educativas, fiscales, Registro Civil, hospitales), las cuales pretenden organizar la sociedad en sincronía con los «avances» de la modernidad.

Semejante modelo determina la segregación de la religión a los espacios privados o a los márgenes del discurso cívico (suerte de barbarie residual digerida por el relato del folclore), que en el correr de las décadas producirá cierta clase de ceguera del Estado frente a las prácticas y necesidades religiosas de la población ${ }^{2}$. A escala general, sopesando la oposición racionalismo/espiritualismo, consideramos que es recientemente con la llegada de los años ochenta, en el contexto de reapertura democrática tras una década de dictadura militar, que comienzan a producirse fisuras significativas en el imaginario racionalista y su marco de unidad cultural ${ }^{3}$. La proliferación en cadena de los flujos desterritorializados asociados a la globalización determinan una distribución nueva de los mismo y lo otro en términos de identidad y pertenencia del sujeto social. Terreno fértil para la proliferación de una amplia gama de Nuevos Movimientos Religiosos ${ }^{4}$, para la configuración de un mercado de bienes simbólico-religiosos ${ }^{5}$. Una

\footnotetext{
${ }^{1}$ Esta lucha ideológica es retratada por A. Ardao, Espiritualismo y Positivismo en el Uruguay, Editorial Universidad de la República, Montevideo, 1968.

${ }^{2}$ Cfr. N. Guigou, A naçâo laica: religiâo civil e mito-praxis no Uruguay, La Gotera, Montevideo, 2003; G. Menéndez, «Crisis del Uruguay laico-racionalista». Cuadernos del CLAEH, n 78-79 (1997), pp. $243-258$.

${ }^{3}$ N. Da Costa, G. Kerber, P. Mieres, Creencias y religiones. La religiosidad de los montevideanos a fin de milenio, Trilce, Montevideo, 1996.

${ }^{4}$ R. Bayce, «Uruguay hoy: la explicable explosión de religiones y sectas», Cuadernos de Marcha, N68 (1992); J. E. Elizaga (1989) Las sectas y las nuevas religiones a la conquista del Uruguay, Editorial La Llave, Montevideo, 1989; R. Geymonat (Ed.), Las religiones en el Uruguay, Ediciones La Gotera, Montevideo, 2004; N. Da Costa, Religión y sociedad en el Uruguay del siglo XXI, CLAEH, CUM, Montevideo, 2003 ; A. Frigerio (Ed.) Ciencias sociales y religión en el cono sur, Centro Editor de América Latina Buenos Aires, 1993.

${ }^{5}$ Al margen de estos nuevos movimientos pero como síntoma del cambio de contexto, cabe recordar la polémica en torno a la instalación de un monumento católico en una de las principales arterias de circulación del centro de Montevideo. La visita del Papa Juan Pablo II en 1987 y, en su homenaje, la instalación de la «Cruz del Papa», hizo surgir de forma manifiesta ideologías que se encontraban latentes en el imaginario uruguayo. Se inicia nuevamente el debate sobre la laicidad del gobierno y de los espacios públicos. El problema desencadenante consistía en dejar o no la Cruz en el espacio público, lo cual implicaba el manejo de la relación del espacio religioso (y principalmente de la Iglesia) con el Estado, los valores nacionales en torno a la lacidad, así como nuevos problemas como la creciente pluralidad y fragmentación de las subjetividad. En torno al hecho viejos debates cristalizaron en nuevas resoluciones, problemas que fluían dispersa y reprimidamente en el imaginario colectivo se inscribieron nuevamente en la superficie del discurso público.
} 
«nebulosa místico-esotérica» ${ }^{6}$ que comprende diversos conjuntos de símbolos y creencias (New Age, orientalismo, psicología transpersonal, Yoga, indigenismo, etc.) en un rizoma de intercambio y comercio «espiritual» donde no son de extrañar orginales combinaciones de símbolos, prácticas y cosmologías?

Este contexto puede considerarse un terreno fértil para el asentamiento de las primeras escuelas budistas en Uruguay, principalmente las relacionadas al budismo tibetano y las corrientes Zen Japonesas ${ }^{8}$. El propósito de este artículo es retratar en pinceladas gruesas el perfil del budismo en Uruguay, haciendo una breve descripción de las escuelas que han llegado a nuestro país. En un trabajo anterior dos de los autores ${ }^{9}$ intentaron una aproximación fenomenológica al rito y las técnicas de conocimiento de los grupos budistas contactados durante el trabajo de campo, en cuyas actividades participamos durante algún tiempo. Esta vez la propuesta es posicionarse en una perspectiva más distanciada, ofreciendo al lector elementos básicos que sirvan para comprender la dialéctica racionalismo/espiritualismo en el devenir de los uruguayos.

\section{TRAZOS DE UNA GENEALOGÍA}

En Uruguay encontramos actualmente varias escuelas budistas, de un modo u otro emparenetadas con tradiciones tibetanas o japonesas. En el primer caso tenemos dos escuelas, una se conoce como Diamond Way (linaje de la tradición Kagyüpa) y la otra como Chagdud Gonpa (linaje Ningmapa). Luego tenemos escuelas vinculadas a budismo de origen japonés: por un lado la Soka Gakkai y la Nichiren Shu (linajes cuya memoria de continuidad se remonta al monje Nichiren), y por otro lado la Asociación Zen del Uruguay (AZU) y el Centro Zen de Montevideo (CZM), ambos emparentados con la tradición soto del budismo Zen.

Desde la antropología religiosa podemos afirmar que en todos estos casos se actualiza el mismo mito fundacional del Buddha, cuyos mensajes cruciales son:

I. Que la vida es sufrimiento (en sánscrito sarvam dukham o «todo es dolor»). La premisa no es exclusiva del budismo sino que es central en la filosofía de la India postupanishadica $^{10}$.

\footnotetext{
${ }^{6}$ F. Champion, «Persona religiosa fluctuante, eclecticismo y sincretismos», En: J. Delumeau, (ed.), El hecho religioso. Enciclopedia de las grandes religiones, Alianza Editorial, Madrid, 1995

7 «En el universo 'fluído', móvil, del creer moderno, liberado de la tutela de las instituciones totales del creer, todos los símbolos son, pues, intercambiables, combinables, y pueden trasponerse los unos en los otros. Todos los sincretismos son posibles, todos los nuevos empleos son imaginables», D. Hervieu-Léger, La Religión, Hilo de Memoria, Herder Editorial, Barcelona, 2005, p. 127.

${ }^{8}$ El caso de las escuelas Nichiren será un tanto distinto, como veremos luego.

${ }^{9}$ I. Apud, M. Clara, «Las tradiciones budistas en Uruguay: miradas antropológicas sobre los caminos de la iluminación», Publicado el 3 de agosto del 2005 en: http://letrasuruguay.espaciolatino.com/apud/tradicio nes budistas.htm; I. Apud, M. Clara, «Representaciones del cuerpo y prácticas terapéuticas en las escuelas budistas del Uruguay». IV Jornadas de Investigación en Antropología Social, Instituto de Ciencias Antropológicas, Facultad de Filosofía y Letras, Universidad de Buenos Aires, 2006.

${ }^{10}$ Los Upanishads son textos sagrados provenientes de la tradición oral de la India. Se remontan al 800 a. C. y fijan el canon cosmológico hindú, a través de nociones como las de samsara (transmigración), karman (causa y efecto), y moksa (liberación).
} 
II. Que la causa del sufrimiento es el deseo. Se trata de la tríada dolor-deseo-ignorancia (en sánscrito dukkha, kama y avidya respectivamente). Estos tres elementos, unidos con la idea del ciclo de reencarnaciones (samsara) y de la causalidad universal (karma), constituyen la red conceptual que soporta todo el pensamiento postupanishádico, dentro del cual se incluye el budismo en tanto solución heterodoxa.

III. Que, si logramos extinguir el deseo despertamos de las ilusiones mundanas para morar en lo eterno y verdadero, mediante la afirmación absoluta de la impermanencia que nos lleva a la disolución del ego (an-atman). Esta hazaña se conoce como iluminación (nirvana).

Asimismo, los referidos linajes de creyentes ${ }^{11}$ provenientes de distintas tradiciones budistas (Vajrayana, Nichiren y Zen) se encuentran genealógicamente emparentados con el Gran Vehículo (Mahayana), que supone históricamente una ruptura con el budismo del Pequeño Vehículo (Hinayana: corrientes antiguas que siguen un modelo monástico «endogámico» de las enseñanzas) ${ }^{12}$. El tronco común del Gran Vehículo instaura en el budismo una vocación misionera ecuménica, el tema de la intuición o gnosis extática del vacío, el cultivo de la gran compasión (mahakaruna) y el interés por articular el punto de vista religioso a los hechos ordinarios del mundo profano, ya sea desde la concepción de deidades o héroes que conjugan figurativamente ambas dimensiones, o desde una especie de deconstrucción de las facultades cognitivas por medio de la meditación abstracta.

\section{BUDISMO TIBETANO}

El Vajrayana o Camino del Diamante es una vertiente budista de amplia difusión en el Tibet, que reúne tanto las enseñanzas básicas de Buda y del Gran Vehículo como influencias tántricas ${ }^{13}$, por ejemplo el manejo de las energías cósmicas y fisiológicas, así como su relación con los aspectos sexuales (masculino-femenino) con los cuales el practicante se identifica durante la meditación. Estas prácticas son acompañadas por

\footnotetext{
11 Tomamos la noción «linaje de creyentes» de la socióloga francesa Hervieu-Leger. Para la autora no existe religión si no hay autoridad de una tradición en el apoyo del creer. «En esta perspectiva, llamaremos 'religiosa' toda forma de creer que se justifique completamente a través de la inscripción que reivindica en un linaje creyente» (D. Hervieu-Léger, La Religión, Hilo de Memoria, Herder Editorial, Barcelona, 2005, p. 137). Para ello se remarcan tres elementos centrales: expresión del creer, memoria de la continuidad, legitimación tradicional. Se trata de la creencia en la continuidad de un linaje de creyentes que trasciende la historia.

${ }^{12}$ F. Tola, C. Dragonetti, Budismo mahayana, Kier, Buenos Aires, 1980; E. Conze, E. El budismo, Fondo de Cultura Económica, México, 1978.

${ }^{13}$ Según E. Conze, El budismo, Fondo de Cultura Económico, México, 1978, pp. 243-275, el tantrismo asquiere su popularidad durante los siglos VI y VII d.C. Se trataría de una adaptación sincrética de la doctrina budista con creencias populares más cercanas al hinduismo en boga, específicamente la devoción (bhakti) a dioses como Shiva y sus consortes. Se basan en escrituras denominadas tantras, así como complejos sistemas de meditación con ricos simbolismos, rituales y fórmulas mágicas. Entre estas escuelas tántricas, adquiere notoria popularidad el Vajrayana, fundada por una sucesión de maestros del extremo norte de la India que atraviesa Nepal hacia el Tibet.
} 
una gran variedad de mudras, mantras y mandalas ${ }^{14}$, de una elaboración y riqueza muy original y de acuerdo a deidades, seres espirituales y bodhisattvas de gran popularidad.

El budismo es introducido en el siglo VIII, a través de la mítica figura del sabio Padmasambhava y la posterior construcción de una gran cantidad de monasterios. Se funda la escuela que posteriormente se denominará Ningmapa o Escuela de los Antiguos, seguidores de la doctrina del dzogh'en (gran perfección). En el siglo X se produce la segunda gran oleada budista en la región: se traducen textos y se instauran nuevas reformas, especialmente con la llegada del gran maestro tantrista Atisa, quien fundaría la escuela Kadmapa (enseñanza oral) que, aunque no sobrevivió, produjo restauraciones decisivas, entre ellas la instauración del gurú o $l a m a^{15}$. En el siglo XI surgen la escuela Kagyüpa o [de la] Tradición Oral, y la Sakyapa, o Tierra Gris, debido al nombre del monasterio en el que se funda. También es en esta época en la que se traduce al tibetano gran cantidad de textos budistas con el nombre de Kanjur y Tanjur. En el siglo XIV surge la escuela de los Gelugpa o Escuela de los Virtuosos, que continúan la línea de los Kadmapa (en oposición a los Antiguos o Ningmapa). Sostienen un método de iluminación progresiva o Lam-Rim, que puede tardar varias vidas en ser realizado. A partir del siglo XVII y con la institución del Dalai Lama, esta escuela asume el control del país.

\subsection{DIAMOND WAY}

La escuela tibetana Camino del diamante Camino del Diamante es una organización budista internacional con amplia difusión en Europa y más de 500 centros en todo el mundo. Perteneciente al linaje budista de los Kagyupa, su fundador es el danés Lama Ole Nydahl, guía de los practicantes uruguayos en este linaje.

El budismo tibetano recibe su primer gran impulso de difusión a nivel popular en occidente a través de Lama Ole. Nacido en Dinamarca en 1941, realiza servicio militar y la carrera de filosofía en la Universidad de Copenhague. En plena ola psicodélica de los años sesenta escribe su tesis de doctorado sobre Las puertas de la Percepción de Aldous Huxley, mientras experimenta con LSD y mezcalina ${ }^{16}$. En ese plan comienza a interesarse por la filosofía budista; viaja con su esposa a Nepal y consigue ser instruido por el XVI Karmapa (máxima autoridad del linaje kagyupa ${ }^{17}$ )

\footnotetext{
${ }^{14}$ Figuras de poder que se realizan con la postura de las manos, con la voz y con representaciones visuales icónicas, respectivamente.

${ }^{15}$ La figura del lama es un elemento central en el budismo tibetano. Actúa como instructor (el discípulo debe tener plena fe en su condición espiritual) y protector público (mediante distintas prácticas rituales así como la recitación de textos sagrados como el Bardo Thödol o Libro de los muertos). Dentro de los lamas encontramos el título de Rimpoch'e o grandemente precioso, o títulos más importantes como el de Dalai Lama en los Gelugpa, o el Karmapa de los Kagyüpa, ambos considerados emanaciones del Boddhisattva Avalokitesvara.

${ }^{16}$ O. Nydhal, Las cosas como son, Garuad Editorial, Colombia, 2003.

${ }^{17} \mathrm{La}$ institución del Karmapa es la primera en incorporar la noción de tulku o «cuerpo de transformación», que implica la decisión del lama de reencarnar nuevamente en beneficio de su escuela. Surge por primera vez en el siglo XIII, con la reencarnación del primer Karmapa Dusum Khyenpa en el segundo Karmapa Karma Pakshi.
} 
en los altos Himalayas. En 1972 funda en Copenhague el primer centro KarmaKagyü europeo.

Su llegada a Uruguay es a través de un practicante de medicina China que viaja en 1997 a un seminario en Venezuela y conoce por casualidad -o causalidad desde la concepción «nativa»- las enseñanzas de la Karma Kagyü. Se da en ese momento un gran intercambio de libros, ideas e intereses, con los que vuelve al Uruguay para continuar estudiando. Posteriormente funda el Centro Budista Camino del Diamante en Montevideo, generando nuevos adeptos para posteriormente fundar el Centro $\mathrm{Bu}-$ dista Camino del Diamante en la Ciudad de la Costa (Lagomar). Actualmente además de estos dos centros, los Kagyupa cuentan con un centro de retiros en la sierra de Minas en Lavalleja llamado Karma Dechen Ling (tierra del mas alto gozo) donde se organizan cursos y actividades relacionadas con el linaje a través de un sistema de traveling teachers (maestros viajeros, estudiantes avanzados de Lama Ole de diferentes partes del mundo) y las clásicas visitas anuales de Lama Ole.

\subsection{LA CHAGDUD GONPA}

De tradición Ningmapa, la Chagdud Gonpa es fundada por Chagdud Tulku Rinpoche (1930-2002), monje formado integramente en el Tibet y propulsor de la difusión del Vajrayana en Estados Unidos, Europa, Rusia, Corea, Hong Kong, Australia y América del Sur. En el caso latinoamericano comienza sus enseñanzas en el año 1991 en Brasil, fundando en 1994 el Khadro Ling, principal centro de estudios de su escuela a nivel mundial. Luego de su fallecimiento en el 2002 la dirección queda a cargo de su esposa Chagdud Khadro.

La Chagdud Gonpa tiene su origen en el siglo XV d.C. cuando Sherab Gyaltsan, primer encarnación de Chagdud Tulku Rinpoche, construye un monasterio en la provincia de Nywgrong, en el Tibet oriental. Gonpa, en tibetano «estar calmo», es la expresión utilizada para nombrar el monasterio. Chag dud o «nudo de hierro» hace referencia a una hazaña prodigiosa de Sherab Gyaltsan: éste, con la sola ayuda de sus dos manos, era capaz de doblar espadas hasta hacerles un nudo, lo cual se convirtió de allí en más en prueba de legitimación que debían de mostrar las sucesivas reencarnaciones del Rinpoche, entre ellas su decimocuarta y última reencarnación (19302002) quien, según sus estudiantes, ha demostrado reiteradas veces poseer la habilidad de doblar espadas de hierro ${ }^{18}$. Fue reconocido como la reencarnación del anterior Chagdud a los tres años de edad, siendo instruído por altos maestros en el monasterio Temphel Gonpa hasta los once años. En 1959, y al igual que una gran cantidad de autoridades religiosas tibetanas, comienza su exilio del Tibet hacia la India, donde transita por varios campos de refugiados, ayudando como «médico». A fines de los setenta viaja a Nepal, donde se realizan grandes encuentros entre las distintas eminencias del budismo tibetano. Es en esta época que conoce a una mujer

${ }^{18}$ Chagdud Tulku Rinpoche, Puertas de la práctica budista. Ed. Chagdud Gonpa, Tres Coroas, 2004. 
occidental norteamericana, Jane Tromge, con la que contrae matrimonio en 1979. Jane seguirá las enseñanzas de Chagdud. En 1996 es reconocida como Lama Chagdud Khadro, acompañando a su esposo y maestro en la enseñanza del dharma durante ventitres años.

Su casamiento con Chagdud Khadro permite la llegada de esta escuela a occidente, al establecerse ambos en Oregón, Estados Unidos. En 1983 se crea la Chagdud Gonpa Foundation y en poco tiempo adquiere fama y reconocimiento. Es en 1992 que recibe su primera invitación para enseñar en Brasil y en 1994 se establece un núcleo en Tres Coroas, estado de Rio Grande do Sul. Allí se construye el primer templo tibetano tradicional, el Khadro Ling (en tibetano «Lugar de las dakinis»), epicentro de la difusión tanto en Brasil (Fortaleza, Rio de Janeiro, Curitiba, San Paulo, Brasilia, Florianópolis, etc.) como en Chile, Argentina y Uruguay.

En 1996 Chagdud Tulku Rinpoche y Chagdud Khadro son invitados a Uruguay. La actividad es promovida por un grupo de uruguayos que, interesados en el budismo, deciden comenzar la práctica. También comienzan las visitas y conferencias de Chagdud Rinpoche quien, al observar el marcado interés de estos estudiantes, decide visitar nuestro país en reiteradas ocasiones, tanto en Montevideo como en el interior. En el año 2000, uno de los estudiantes de la escuela dona a Rinpoche un terreno en Aguas Blancas, Lavalleja, donde se instala el primer centro de retiros de la Chagdud Gonpa Hispanoamérica. Actualmente el monasterio se encuentra en plena actividad.

En la noche del 4 de noviembre del 2002, Chagdud Tulku Rinpoche fallece consecuencia de un ataque cardíaco, quedando Chagdud Khadro a cargo de los centros latinoamericanos. La muerte de Chagdud Rinpoché es rodeada de una trama sobrenatural: su cuerpo no sufre los procesos naturales de descompsición, un arcoiris surge misteriosamente durante su traslado a Nepal, los alumnos del lama experimentan sueños maravillosos. Actualmente ellos esperan y realizan plegarias que anuncian su decimoquinta reencarnación, todavía no manifestada.

\section{BUDISMO ZEN}

Existen varias maneras posibles de confeccionar el árbol genealógico del Ch'an/Zen en China y en Japón, o sea, de analizar las conexiones filiales por donde pasa y se bifurca la transmisión de maestro a discípulo. Al seguir la llamada «línea de sangre», el genealogista no debe pasar por alto la sobredeterminación que achaca a los nombres propios (una serie continua o discontinua de ellos) en tanto símbolos, no de un significado oculto o esotérico, sino de una variación respecto a la herencia unívoca del Dharma: de cierta reforma que inicia una nueva escuela, de un temperamento peculiar o de un estilo de sabiduría. El tronco común se alarga a través de casi doscientos años, entre el arribo de Bodhidharma desde la India (? -543) y la heroica sucesión del sexto patriarca Hui-neng (638-714). Luego empezamos a constatar variaciones en la manera de hacer pasar la línea, si bien siguiendo las nervaduras, a partir del siglo 
IX, de las llamadas «Siete Casas» del budismo Ch'an: Escuela Sôto, Escuela Rinzai, Escuela Ôryo, Escuela Yôgy (las dos últimas como subdivisiones de la Rinzai), Escuela Ummon, Escuela Hôgen y Escuela Iggyô ${ }^{19}$.

Los chinos conocieron el budismo en el momento que Occidente entró en la era cristiana. No caben dudas de su retroalimentación con el taoísmo, especialmente con el llamado «taoísmo filosófico» (el quietismo naturalista de Lao-tse, Chuang-tsu, y Lie-zi) cuyas coincidencias o encuentros con el anti-conceptualismo radical de Nagarjuna en torno al principio de la vacuidad (Tao para los primeros, Sûnyata para este último) determinarán posteriormente las características estructurales del Ch'an/Zen en su diversidad de manifestaciones históricas. No obstante, será recientemente durante la dinastía Tang (618-907, período en el que el imperio chino vive su apogeo civilizador) que, en la medida que se desarrollan las traducciones y comentarios de los sutras canónicos y los sastras mahayánicos, el budismo se convierte en el poder espiritual hegemónico, frente a la pobre resistencia de la ortodoxia confuciana. Este período se divide en dos etapas, en la primera de las cuales domina un budismo «escolastizado», que se concentra en la corte y en las academias formativas de las élites políticas e intelectuales; en la segunda etapa (hacia el siglo VIII) China recibe la impetuosa penetración del llamado budismo «esotérico», asociado a las doctrinas tántricas, preñado de ritos suntuosos, saberes alegóricos y magia. Precisamente, «...contra esta doble oleada de indianización se levanta la escuela Ch'an, resurgimiento de la vieja mistica taoísta, desviación china del budismo que acabará por fundirse con un movimiento de reforma del confucionismo cuyo triunfo marcará la época de los Sung» ${ }^{20}$.

Dentro de la línea de filiación Ts'ao-tung/Sôto -que es de la que principalmente nos estamos ocupando aquí- hay cuatrocientos años subtendidos entre su germinación en la China de la era Tang y su maduración en el Japón de la época Kamakura. Con la extinción de los filums Hôgen, Ummon e Iggyô y el paulatino debilitamiento de las subescuelas Ôryo y Yôgy, el terreno del Zen se divide con nitidez entre las vertientes Sôto y Rinzai, una bajo la bandera del silencio (mokusho-zen) y el otro de la palabra (kanna-zen) en lo que respecta al paradigma de enseñanza.

A la hora de examinar el desenvolvimiento del Zen en Europa debemos mencionar como hito fundacional la llegada a Paris del japonés Taisen Deshimaru en 1967, puesto que de su fervor misionero surgirán la mayoría de los grupos que continuaron en el viejo continente la tradición Sôto hasta nuestros días ${ }^{21}$. Deshimaru se convirtió en discípulo del Roshi Kodo Sawaki en 1930, aunque siguió manteniendo una agitada vida seglar como maestro de escuela, estudiante universitario, empleado de grandes compañías, empresario, político, funcionario del gobierno y asistente social. Fi-

19 D. T. Suzuki, Ensayos sobre budismo Zen. Primera serie, Kier, Buenos Aires, 1995; D. T. Suzuki, Ensayos sobre budismo Zen. Segunda serie, Kier, Buenos Aires, 1986; D. T. Suzuki, Ensayos sobre budismo Zen. Tercera serie, Kier, Buenos Aires, 1989.

${ }^{20}$ P. Demieville, «El budismo chino», en: H. Puech (ed.), Historia de las religiones, vol. 4, Siglo XXI, México, 1986, p. 342.

${ }^{21}$ T. Deschimaru, Autobiografía de un monje zen, Kier, Buenos Aires, 1986. 
nalmente, en 1965 recibe la ordenación de monje con Sawaki y dirige por dos años el dojo de Shoyogi en Tokio, hasta que un grupo de macrobióticos franceses lo invitan a un viaje de divulgación del zen en su país. En los años '70 su misión cobra vuelo, especialmente después que recibe la trasmisión del Dharma y le nombran Kaikyosokan, responsable del Sôto-Zen para un país o continente. Deshimaru pasa enseguida a fundar la Asociación Zen Internacional (AZI), con el objetivo de difundir en toda Europa la práctica y la enseñanza de su tradición religiosa. La AZI pronto se convertirá en un poderoso organismo de coordinación en el continente (hoy representa a más de 200 dojos). En 1979 Deshimaru funda en Francia el Tai Sei Bukkyo Dai Ichi Dojo («primer gran dojo de occidente para la enseñanza del Buda»), popularmente conocido como La Gendronniére por ser el nombre de la finca donde se alza (en la Sologne, valle del Loira). En este centro se formarán las sucesivas generaciones de discípulos, que tras la muerte de Deshimaru en 1982 siguen propagando su linaje por las principales capitales europeas y en varios países de América.

Cuando Deshimaru Roshi fallece, deja una carta en la que nombra tres sucesores, entre los que se cuenta Estefan Thibaut o Kosen (nombre de ordenación), quien por diferencias con los otros dirigentes se separa poco tiempo después de la Asociación Zen Internacional y funda su propia Kosen Sangha. A principios de los '80 un grupo de argentinos que se encontraba viajando por Europa toma contacto con esta sangha $\mathrm{y}$, a su regreso, deciden establecer en Buenos Aires un espacio de meditación ${ }^{22}$. El maestro Kosen comienza entonces a realizar visitas periódicas, supervisando la creación de la nueva comunidad. En el transcurso de la década, mientras crece el dojo de Buenos Aires, van apareciendo otros espacios en Rosario, Mar del Plata, Esquel, Trelew, La Plata y Córdoba, a la par que van formándose grupos asociados a la Kosen Sangha en otros países de América (Cuba, Brasil, Chile, Bolivia y Venezuela). A este crecimiento responde la fundación de una Asociación Zen de América Latina, cuya obra más importante hasta la fecha (aparte de su constante función coordinativa) fue el establecimiento en 1997 del monasterio Shobogenji en Córdoba (en el cerro Uritoco, Capilla del Monte), donde no sólo residen monjes en forma permanente, sino que abre sus puertas todo el año para la visita del público y, lo que constituye su principal objetivo, sirve para la realización del «campo de verano», que se trata de un retiro tipo sesshin donde los discípulos americanos toman contacto con el maestro Kosen para recibir sus enseñanzas, vivir en comunidad y meditar intensivamente a lo largo de un mes.

En el caso de Uuguay, a fines de los ochenta un practicante de Aikido vuelve de Japón y comienza a enseñar la práctica del zazen. Se trata de un personaje carismático, psicólogo e instructor de Tai-Chi en la Fundación Sino-Uruguaya. En 1997 funda la Asociación Zen del Uruguay (AZU), no como organización religiosa, sino como organización civil. Sigue las enseñanzas de Deshimaru, si bien no se inscribe ni en la AZI, ni en organización internacional alguna. Se trata más bien de un divulgador,

\footnotetext{
${ }^{22}$ Cfr. C. Carini, «El budismo zen en Argentina», publicado en 2004 en Congreso Virtual de Antropología NAYA, http://www.naya.org.ar.
} 
que asume el compromiso de orientar la meditación de forma totalmente laica, si bien su «magnetismo» personal, la incorporación de una gama filosófica y terapéutica de «saberes orientales» y su formación profesional como psicólogo lo convierten en una especie de líder espiritual. Será reconocido como «monje», representante de Deshimaru y pensador budista original, al cual se le ordenan discípulos y seguidores. Sin embargo con el tiempo comienzan a generarse equívocos en torno a su legitimidad y en la segunda mitad de la década de los noventa comienza la desarticulación de éste grupo de practicantes. En ese momento, algunos ex miembros asisten a un retiro en el mencionado dojo de Buenos Aires bajo la orientación de dos discípulos de Kosen, y desde allí comienza a tramarse la creación del Centro Zen de Montevideo (CZM), como institución separada e independiente de la facción que posteriormente constituirá la AZU. Ésta última -completamente escindida de su «maestro» fundador- decide escribir una carta al monasterio Busshin-ji de San Pablo, sede central de la actividad misionera de la escuela Sôto para América del Sur, y pionera en la tarea de difundir la práctica Zen en América del $\operatorname{Sur}^{23}$. Es a través Roshi Daigyo Moriyama -responsable general del monasterio así como Kaikyosokan de la Sôto Zen en América del Sur- que la AZU establece lazos institucionales a nivel internacional.

En los últimos años, el número promedio de practicantes de la AZU ha sido de unas diez a quince personas, mientras que el del CZM de cinco a diez. Por regla general, el grupo de practicantes se divide de acuerdo a su concurrencia por la mañana o por la tarde, superando rara vez las 5 personas cada uno. Por lo menos una vez al mes, se utiliza un día del fin de semana para «reuniones de rakusu», o sea de los practicantes ordenados en forma laica como discípulos del maestro, los cuales asumen los «preceptos del bodhisattva ${ }^{24}$. Suele suceder que semejantes reuniones den pie a actividades de entrenamiento y difusión (inclusive «mini-sesshin»), o bien que se aprovechen para el planteo de cuestiones institucionales.

El corazón de la práctica soto zen es el zazen. En relación a la pauta que diseña Dôgen ${ }^{25}$, destinada a especificar la modalidad central de entrenamiento, antes que nada y sin excepción, el iniciado practica zazen shikantaza. Shikan (nada sino) ta (adecuadamente) $z a$ (sentarse) ${ }^{26}$ : «Pasar el tiempo sentado correctamente sin nada que ganar ni nada que comprender es la Vía de los patriarcas ${ }^{27}$. No significa dejar la mente en blanco, ni se cree ni considera esta posibilidad. Sólo se trata de mantener la postura, la respiración y la conciencia justas, equilibradamente, en calma. Esto último despierta el mayor número de interrogantes, pues requiere «dejar de pensar» (fu shiryo:

\footnotetext{
${ }^{23}$ No es extraño que la primera misión oficial de la escuela Sôto por Sudamérica haya sido en Brasil, país que, por lejos, cuenta con la mayor inmigración japonesa dentro del continente «Brasil tiene en la actualidad una población de 130 millones de habitantes; de ellos, 1.160.000 son japoneses o descendientes» (T. Yanaguida, M. D. Rodriguez, Japoneses en América, Mapfre, Madrid, 1992, p. 237). Este contingente es el más grande de América Latina, secundado en bastante menor medida por el de México, Perú y Argentina.

${ }^{24}$ Morishama Roshi, Primeiros passos no Zen, Bodigaya, Porto Alegre, 2001.

25 Padre del budismo soto japonés (1200-1253).

${ }^{26}$ S. Schumacher, G. Woerner, Diccionario de la Sabiduría Oriental, Paidós, Barcelona, 1993, p. 324.

${ }^{27}$ E. Dogen, Shôbôgenzô Zuimonki, Miraguano, Madrid, 1998, p. 134.
} 
no-pensamiento). No es hacer tabula rasa, sino cortar la intención de pensar, la identificación y el apego a los pensamientos. Pensamientos, voces e imágenes interiores van y vienen como nubes que evocan identidades imaginadas; "sin atracción ni rechazo», simplemente pasan. En este plan, el proceso del zazen es la reducción al absurdo del Yo en pos de la inmanencia interior-exterior.

\section{BUDISMO NICHIREN}

El budismo Nichiren es fundado por Nichirén en el siglo XIII, enmarcado en un período histórico de quiebre con el budismo sacerdotal y de élite, y el desarrollo de concepciones pragmáticas y porpulares, a través de reformadores que intentan abrir el acceso a la salvación de las capas sociales mas humildes de Japón ${ }^{28}$. Independientemente de la organización y la nación a la que pertenezcan, los seguidores de Nichiren deben recitar diariamente el mantra Namu-Myoho-Renge-Kyo y algunos capítulos del Sutra del Loto en su idioma original (japonés).

El objeto de veneración es el Gohonzon: un mandala confeccionado por el propio Nichiren, cuyo soporte es un pergamino de papel de arroz en el que están escritos con tinta china, caligráficamente, los ideogramas de la fórmula Namu-Myoho-Renge-Kyo, y escoltándolos a derecha e izquierda (todo verticalmente) los nombres de algunos budas y bodhisattvas. A diferencia de los famosos mandalas del budismo tibetano (figurativos y más o menos abiertos a la interpretación a partir de una compleja gama de alegorías), el mandala de Nichiren es abstracto, único y poco dado a la absorción contemplativa. Su fuerza simbólica viene más bien del singularísimo acontecimiento de la inscripción de la Ley en una Tabla por parte del mesías. El Gohonzon original (escrito de puño y letra por Nichiren el 12 de Octubre de 1279) se encuentra en el templo principal de la Nichiren Shoshu y representa, junto al daimoku - acto de enunciación del Namu Ryoho Renge Kyo-y al propio santuario, uno de los «Tres Misterios» (o «Tres Grandes Leyes Secretas») que algún día, de acuerdo a la teleología mesiánica del kosen rufu, habrá de ser venerado por toda la humanidad.

\subsection{SOKA GAKKAI}

En 1930 Tsunesaburo Makiguchi y Josei Toda fundan en Tokio la Soka Kyoiku Gakkai, «Sociedad Pedagógica para la Creación de Valores», una asociación civil constituida por educadores e intelectuales comprometidos en la reforma del sistema educativo japonés, a través de un método pedagógico enfocado a la formación de ciudadanos con sentido de la responsabilidad, no sólo como agentes históricos de su país, sino como miembros de una humanidad que no reconoce fronteras. Estas ideas iban

\footnotetext{
${ }^{28}$ Cfr. D. Ikeda, D., Saito, K. Diálogos sobre la Religion Humanística. El mundo de los escritos de Nichiren Daishonin, USGI, Montevideo, 2002.
} 
en contra de las disposiciones educativas del gobierno militarista japonés (movilizado hacia el imperialismo y la guerra), que además eran sometidas explícitamente a la crítica. En sus comienzos la Soka Kyoiku Gakkai emprendió movilizaciones para denunciar las manipulaciones nacionalistas del Estado sobre la enseñanza escolar y religiosa; diez años más tarde fue proscrita.

Algunos de sus miembros fueron detenidos y Makiguchi y Toda encarcelados bajo la acusación de «criminales ideológicos». Makiguchi muere martirizado en prisión, en 1944. Poco antes de la rendición militar de Japón (Julio de 1945) Josei Toda fue liberado y se pone a trabajar inmediatamente en la reconstrucción de la Soka, adhiriendo formalmente a la Nichiren Shoshu (la más importante iglesia de Nichiren en Japón) a modo de brazo laico. En pocas palabras, Toda se consagró a ampliar el campo de actividades de la organización (ahora rebautizada simplemente como Soka Gakkai, «Sociedad para la creación de valores»), poniendo gran énfasis en el estudio y la propagación del budismo. En base al lema de la «revolución humana» ${ }^{29}$ enseñaba que, a través de la práctica budista y de una transformación motivada en el interior del individuo, todas las personas podrían cambiar su destino para bien, lo cual tuvo una poderosa resonancia entre mucha gente que sufría de pobreza, enfermedad y otros retos en el caos de la posguerra en Japón.

Toda fallece en 1958; en 1960 asume como tercer presidente Daisaku Ikeda. Se propone seguir adelante con el programa para «una paz mundial» ${ }^{30}$, sensiblemente urgente en el contexto de la amenaza atómica durante la Guerra Fría. Sus primeras iniciativas están relacionadas a la abolición de las armas nucleares, mediante campañas dirigidas a las Naciones Unidades. También brinda conferencias en varias partes del mundo, logrando entrevistarse con líderes políticos e intelectuales eminentes y promoviendo intercambios culturales. En 1962, la Soka entra en la escena política japonesa de manera formal, con la fundación del partido Komeito («Gobierno Limpio») y, a finales de esta década, detrás de los socialistas y de los liberales, se convierte en la tercera fuerza política de Japón.

No obstante, lo que viene afirmándose con el correr de los años es la dimensión internacional del gran objetivo de Nichiren y de la Soka: el kosen rufu (literalmente: «declarar y propagar ampliamente»), que consiste en la aceptación internacional de la fe en el «auténtico budismo» expuesto en el Sutra del Loto, único cimiento legítimo para la construcción de «una paz duradera». En la medida que los responsables nipones sofistican su organización e Ikeda siembra acuerdos y nuevos centros por todo el planeta, en 1975 la Soka vuelve a rebautizarse, agregándole a su nombre el adjetivo Internacional (SGI). Al mismo tiempo, se produce un gran despliegue institucional, ampliándose su aparato educativo en Japón ${ }^{31}$ (que abarca todos los ciclos) y creando, entre otras fundaciones, la Universidad Soka de los Estados Unidos, el Instituto Toda

\footnotetext{
${ }^{29}$ D. Ikeda, La revolución humana, Vol.1, Emecé, Barcelona, 1990.

${ }^{30}$ D. Ikeda, Una paz duradera, Vol 1, Emecé, Barcelona, 1987; D. Ikeda, Una paz duradera, Vol 2, Emecé, Barcelona, 1991.

${ }^{31}$ D. Ikeda, «Avanzar por el camino de la paz, la cultura y la educación». La raíz del ceibo, n 237 (2004), pp. 6-11.
} 
para la Paz Mundial, la Asociación de conciertos Min-On, el Museo de Arte Fuji en Tokio, el Centro bostoniano de Investigaciones para el siglo XXI, la Casa Literaria Víctor Hugo y el Instituto de Filosofía Oriental. A pesar de su polémica ruptura con la estructura eclesiástica de la Nichiren Shoshu (en 1991), actualmente la SGI se jacta de contar con unos 12 millones de miembros repartidos en 190 países.

Esta organización da sus primeros pasos en Uruguay a mediados de la década de 1960, siendo entonces pionera en la introducción del budismo. En una primera instancia lo hace informalmente, representada por algunas familias de inmigrantes japoneses que traen su «altar de veneración» (gohonzon). Ahora bien, siendo uno de los preceptos de la Soka difundirse en pos del kosen rufu planetario, a medida que los inmigrantes se estabilizan, entablan relaciones de sociabilidad con los nativos y comienzan a hablar el español, transmiten su fe en los entornos que frecuentan y abren las puertas de sus hogares a la gente interesada. El principal agente de la difusión fue el actual director de la SGI uruguaya, Tadashi Tanaka. Vinculado profesionalmente a tareas en el puerto de Montevideo, su hogar en el barrio de la Aduana es el principal referente de las «reuniones de diálogo» y de culto por aquellos años. Los más veteranos entre los miembros que entrevistamos recuerdan un grupo inicial muy reducido, conformado en su mayoría por japoneses, que contaba con materiales de estudio redactados en su lengua natal o en inglés.

A principios de los años ' 70 , de los contactos con los órganos administrativos de la Soka internacional, resulta la institucionalización de una sede en Uruguay. La estrategia de transmisión de la fe en el marco de la familia, y de familia en familia, obtuvo rápidos resultados, tejiéndose paulatinamente una red de «comunidades» en diversos sectores de la capital que en los últimos años se ha extendido a varios departamentos del interior del país. Actualmente, la Soka Gakkai es la institución budista más numerosa en Uruguay. Según cifras de la organización ascienden a 700 familias, lo que arrojaría un total aproximado de 2000 adeptos.

\subsection{LA NICHIREN SHU}

Nichiren-shū es una confederación que reúne un conjunto de escuelas budistas seguidoras de Nichiren, reuniendo más de 5000 templos en el mundo. A diferencia de la Soka, no identifica a Nichiren como una reencarnación del Buda Sakyamuni, sino que lo concibe como la encarnación del Bodhisattva Jogyo, descentramiento que -a diferencia de la Soka o la Nichiren Shoshu- brinda una mayor soltura a la hora de relacionarse con otras órdenes budistas. La propagación de la Soka por todo el mundo, parece haber incentivado a la Nichiren Shu a intensificar su tarea misionera, llegando primero a Estados Unidos y posteriormente a Europa y Sudamérica.

El primer contacto con uruguayos se realiza en el año 2002 a través de una pareja perteneciente a la Soka Gakkai, que comienzan a interesarse a través de internet por la escuela Nichiren Shu. Es a través de la web que se contacta con un miembro español, y posteriormente con las escuelas de Estados Unidos e Italia, que ofrecen apoyo, 
brindándoles materiales de lectura y vinculándolos con la escuela a nivel internacional. A principio del 2003 se contactan con el templo de la Nichiren Shu en Brasil, destinado principalmente a la propagación del dharma entre la comunidad japonesa. Pero es recién con la llegada - ese mismo año- del Rev. Shoyo Tamura, destinado a la propagación de la Nichiren-Shu en Latinoamérica, y la consecuente fundación del templo Hokkekyo ji en San Pablo que comienza una comunicación más fluida y asidua. Es este monje el que les entrega el «Ippen Shutai Omandara», daimoku provisorio que se ofrece a futuros monjes de la escuela. Habrá que esperar al 2006 para que Shoyo Tamura viaje a Montevideo y realice la entrega del Gohonzon a la pareja de practicantes. En ese entonces se habían sumado a la práctica algunas personas interesadas; a su vez, y a través de la escuela uruguaya, la Nichiren Shu logra extenderse hacia Buenos Aires y la Patagonia. En el 2007, el Rev. Dairyo Tomikawa-Supervisor Asistente de la Sede Central de Nichiren Shu en Japón- visita Montevideo y la Patagonia, así como también el nuevo responsable para Sudamérica, el Rev. Eisei Ikenaga, quien se encargará de dirigir y visitar periódicamente a sus discípulos uruguayos. Actualmente, los referentes de la escuela uruguaya son los reverendos Eisei Ikenaga Shonin y Yodo Okuda Shonin, quienes periódicamente visitan nuestro país e imparten enseñanzas. También se realizan conferencias, teleconferencias, talleres, y reuniones con otras escuelas de otros países.

\section{OTROS MOVIMIENTOS RELACIONADOS CON EL BUDISMO}

Dentro de las nuevas formas que asumen las prácticas religiosas orientales en su contacto con occidente encontramos manifestaciones religiosas y sincretismos inéditos, de los que el budismo no es una excepción. Tal es el caso de la Juniper Foundation, institución que hace unos años se dedica a la enseñanza de un budismo secularizado y occidentalizado, construido en la paradoja de un mercado de bienes simbólico-religiosos en el que intenta tener éxito y del que intenta escapar a través del rechazo a la parafernalia de consumo de una otredad exótica.

Juniper es un árbol cuyas variedades se encuentran diseminadas en Europa, Asia y Norteamérica, y que muchas culturas le adjudican propiedades medicinales y purificadoras. Es utilizado como metáfora de la acción realizada por la fundación, que supone adaptar el budismo a occidente, de modo de suprimir aquellas pautas relacionadas al consumo superficial e incentivar aquellas otras que son consideradas como «medulares». La Fundación Juniper se basa en un entrenamiento mental que toma las enseñanzas budistas para el desarrollo personal de sus integrantes. Se trata de una institución que resignifica la práctica y valores budistas de acuerdo a las reglas de juego del mundo occidental moderno, suprimiendo lo más posible las connotaciones religiosas y concibiendo al Buda no como un personaje sagrado, sino como una persona más, con una «gran idea». Dentro de sus objetivos de la fundación se encuentran el cultivo de la calma interna, la claridad mental, así como un método de curación que brinda asistencia a enfermedades y problemas. Si bien dice formar 
parte de la genealogía del linaje tibetano Gelugpa, se eliminan las simbologías iconográficas y aquellos elementos rituales que puedan ser capturados por el fetichismo de consumo de diversidad cultural de occidente. Paradójicamente, este intento de escapar del consumo occidental se redefine en una abstracción filosófica que cumple con los cánones de una cultura occidental intelectual y secularizada. Se incluyen, por ejemplo, textos no tradicionales relacionados con filósofos como Charles Searle, o disciplinas como las neurociencias. La idea es recontextualizar el budismo a los tiempos modernos, transformación justificada bajo el hecho histórico de que la práctica budista se ha transformado a lo largo de su recorrido por las distintas culturas por las que se ha difundido.

La fundación posee tanto una escuela de aprendizaje como una clínica. En la escuela de aprendizaje se enseña un entrenamiento basado en la meditación y el estudio analítico de la mente. La clínica utiliza un método terapéutico que combina medicina y técnicas orientales con avances de las neurociencias, buscando integrar mente y cuerpo en los procesos de curación. Su líder, Segyu Rinpoche, nació en Rio de Janeiro y reside en California. Fue reconocido por el 98avo Gaden Tri Rinpoche como maestro del linaje Segyu de la escuela Gelugpa, así como identificado como tulku de un abad del siglo XIV. Luego de veinte años de práctica decide modernizar la práctica budista, a través de la Fundación Juniper, en el año 2003. Los practicantes uruguayos entran en contacto con Segyu Rinpoche a fines de los años noventa, cuando la modernización de la práctica todavía no había sido realizada definitivamente. Los primeros contectos se realizan gracias a una acaudalada mujer norteamericana que costeaba los gastos para traer al maestro a difundir sus enseñanzas. Luego, y en su ausencia, el grupo de uruguayos tuvo que costear los gastos con sus propios medios. Se formaron tres grupos, dos de ellos en Montevideo, y uno en Maldonado, actualmente disuelto. Son grupos pequeños con un núcleo estable de aproximadamente 10 personas, que meditan tanto individual como colectivamente, así como participan en la traducción de textos.

\section{CONCLUSIONES}

Díficil es pensar la llegada del budismo en Uruguay sin plantearnos la decisiva influencia de un contexto político post-dictadura, una creciente globalización en lo cultural y económico, y la consolidación de un mercado simbólico-religioso relacionado con el desencantamiento del proyecto moderno y de las instituciones religiosas tradicionales. Con excepción de las corrientes Nichiren, -cuya llegada en Uruguay se realiza principalmente a través de familias de inmigrantes japoneses pertenecientes a la Soka Gakkai-, lo que observamos en los demás casos es un interés por formas alternativas de religiosidad, por lo general emparentadas con lo que usualmente se denomina «nebulosa místico-esotérica», y que deriva en contactos con escuelas tradicionales de otras partes del mundo, a través de viajes al exterior y de las nuevas tecnologías de comunicación vinculadas a internet. 
El ideal «emancipatorio» budista plantea notorias diferencias con los ideales de «liberación» secular característicos de nuestro país (emancipación gracias a la progresiva capitalización de conocimiento y recursos, y/o emancipación gracias a la revolución de un orden social opresivo). Con la dictadura militar, estos ideales pierden fuerza y, finalizada ésta, la situación cultural del país acompasa a la de un mundo globalizado y (pos)modernizado. Es allí que comienzan a emerger una pluralidad de opciones religiosas, no tanto vinculadas a proyectos colectivos de gran escala, sino a ofertas individuales de salvación, curación y sentido, que sirven como apoyo frente a la crisis de las instituciones tradicionales. De esta manera, diversas prácticas y creencias tributarias del «orientalismo» y de la denominada «nebulosa místico-esotérica» encuentran campo fértil para su propagación, en relación más que nada a la demanda de un público perteneciente a una clase media desencantada y con cierto «capital cultural» que lo hace propenso a la reflexión intelectual-introspectiva sobre los problemas de corte estrictamente práctico. Encontramos sus primeras manifestaciones en la emergencia dispersa de la oleada «New Age» a principios de los años 80, que actuó determinantemente en el establecimiento del budismo (no obstante este último revele un mayor afán de formalización).

En el caso del budismo, su difusión en Uruguay parece poder ser aceptada en aquellas capas sociales con cierto grado de educación. Su complejidad conceptual así como su alto grado de abstracción en problemas filosóficos (muchas veces comunes a nuestra propia tradición) parecen ser el motivo principal ${ }^{32}$. El budismo ofrecería una solución bastante diferente y paradójica al problema del sufrimiento y del sentido, quizás no lo suficientemente pragmática para las capas «populares», aunque sí muy atractiva para las necesidades existenciales de los sectores con mayor capital cultural. Por un lado tenemos una limpieza kármica, donde lo negativo es extirpado en lo que los budistas consideran su raíz (desde su punto de vista muy peculiar de las relaciones sujeto-objeto). Dicha purificación implica una terapéutica (de gran interés por los adeptos a lo que se denomina «nebulosa místico-esotérica»), aunque sería sólo un aspecto de la solución budista, e inclusive no más que una fase preliminar a la única solución final posible o sea, la iluminación.

Es significativo como la formación de la mayor parte de las escuelas presentes en Uruguay (con excepción de la Soka Gakkai) se da en forma espontánea, sin una estrategia misionera explícita por parte de las organizaciones internacionales que las contienen. La emergencia de cada una de ellas en nuestro país se da bajo el interés de sus futuros practicantes, enmarcados en un interés místico posmoderno que los lleva a contactarse con organizaciones internacionales, gracias a las facilidades de comunicación y movilización geográfica propias de un mundo globalizado. Desde la perspectiva de los propios actores, la reconstrucción del encuentro con el linaje de creyentes implica la sutura de una memoria que establece una continuidad entre los tiempos

\footnotetext{
32 Para una visión mas general de la difusión del budismo en el contexto latinoamericano e internacional cfr. con H. Durt «El desarrollo del Budismo en Occidente: su realidad y significado». Revista de Estudios Budistas, Asociación Latinoamericana de Estudios Budistas, 2.3 (1992), pp. 168-176.
} 
anteriores a la ordenación y el encuentro con la esucela y los maestros con los que se vincula. Se trata de un encuentro propiciado por «vientos kármicos», donde la causalidad por la generación de buenas acciones pasadas -sea en ésta u otras vidas- genera el encuentro con la doctrina. Esta modalidad de difusión no implica un misionerismo activo (como en los pentecostales, por ejemplo) sino una especie de misionerismo receptivo, que escapa de aquellas formas clásicas de lucha simbólica por el reconocimiento dentro del aparato estatal o los medios de comunicación masivos.

Por último, también es interesante observar cómo cada una de las escuelas actúa por separado, muchas veces desconociendo su existencia mutua en el contexto nacional. El budismo no constituye, pues, un «frente común». Tampoco, cada escuela por su lado, viene a publicitar una cosmovisión. Se trata de propuestas independientes, integradas en un mercado de bienes simbólicos de libre competencia, que ofrecen procesos terapéuticos perfectamente compatibles con las exigencias de la vida laica en una cultura fragmentada. 